\title{
Marketing Messages in Continuing Medical Education (CME) Modules on Binge-Eating Disorder (BED)
}

\author{
Jinbyun Jung, BS and Adriane Fugh-Berman, $M D$
}

Background: In 2015, Vyvanse (lisdexamfetamine) became the first Food and Drug Administration (FDA)-approved treatment for binge-eating disorder (BED), a condition first recognized by the DSM-V in 2013. Because pharmaceutical companies use continuing medical education (CME) to help sell drugs, we explored possible bias in CME modules on BED.

Methods: We utilized a qualitative thematic analysis research approach to identify and classify patterns in CME activities focusing on BED.

Results: We identified 27 online CME activities on BED in 2015. All were funded by Shire, which manufactures lisdexamfetamine. Seven of $\mathbf{1 6}$ presenters disclosed financial ties with Shire. Twenty-nine slides recurred in at least 2 CME modules, and 12 slides were repeated in 5 or more modules.

Diagnosis-related themes included: BED is a real, treatable disease; BED is highly prevalent but often missed; BED can occur in anyone; BED results in poor quality of life; many patients with BED are obese; and BED makes losing weight difficult. Treatment-related themes included: lisdexamfetamine is highly effective; topiramate is limited by substantial adverse effects; and other therapeutic options for BED are inferior to lisdexamfetamine because they do not cause weight loss. Although amphetamines can cause addiction, myocardial infarction, stroke, and death, no module mentioned these serious adverse effects.

Conclusions: It seems that CME is being used to promote lisdexamfetamine for weight loss (a contraindicated use) and to highlight benefits of lisdexamfetamine while underplaying the risks. ( $\mathrm{J}$ Am Board Fam Med 2020;33:240-251.)

Keywords: Amphetamines, Binge-Eating Disorder, Continuing Medical Education, Lisdexamfetamine, Quality of Life, Weight Loss

Most physicians are required to take continuing medical education (CME) courses for license renewal, and the quality of information provided affects patient care. Although most CME that physicians view is sponsored by the pharmaceutical

This article was externally peer reviewed.

Submitted 7 April 2019; revised 27 September 2019; accepted 1 October 2019.

From the University of North Carolina Eshelman School of Pharmacy, Chapel Hill, NC (JJ); Department of Pharmacology and Physiology, Georgetown University Medical Center, Washington, DC (AFB).

Funding: None.

Previous Presentations: A portion of this project was presented at the Georgetown University Medical Center PharmedOut conference, Washington, DC, June 15, 2017.

Disclosures: JJ has no conflicts of interest. AFB directs PharmedOut, a Georgetown University Medical Center project that encourages rational prescribing. AFB also has a contract with the George Washington Milken Institute School of Public Health to analyze pharmaceutical industry, very few studies have examined commercial bias in sponsored CME. ${ }^{1}$ The few studies that do exist have found bias in favor of sponsors' drugs,${ }^{2-6}$ raising the question of whether industryfunded CME compromises rather than promotes rational prescribing.

Pharmaceutical companies consider CME important for marketing ${ }^{7}$ and documents disclosed in

marketing data from Washington DC. AFB is also a paid expert witness at the request of plaintiffs in litigation regarding pharmaceutical and medical device marketing practices.

Conflicts of interest: JJ has no conflicts. AFB is a paid expert witness at the request of plaintiffs in litigation regarding pharmaceutical and medical device marketing.

Corresponding author: Adriane Fugh-Berman, MD, Department of Pharmacology and Physiology, Georgetown University Medical Center, 3900 Reservoir Rd. N.W. MedDent SE 402, Washington DC 20057 (E-mail: ajf29@ georgetown.edu). 
litigation emphasize the importance of CME to marketing new drugs or new indications. ${ }^{8-9}$ Bingeeating disorder, first recognized by the DSM-V in 2013, may be an example of a disease created to sell a drug. ${ }^{10}$ A study of conflicts of interest among DSM task force and work group members involved in assessing new diagnoses, including binge-eating disorder, found that conflicts of interest regarding research and nonresearch relationships were common. ${ }^{11}$

Although binge eating is certainly an abnormal behavior that can be a symptom of stress, anxiety, depression, or obsessive-compulsive disorder, little evidence supports treating binge eating as a unique disease. In fact, binge-eating disorder has been cited as an example of pathologization of normative eating patterns. ${ }^{12-16}$ In any case, therapy, especially cognitive behavioral therapy, has been shown to be an effective treatment for binge eating. ${ }^{17-18}$

In January 2015, Vyvanse (lisdexamfetamine) became the first drug approved for binge eating disorder. The drug was already popular; in 2014, lisdexamfetamine generated over a billion dollars of revenue. ${ }^{19}$

Lisdexamfetamine, which is simply dextroamphetamine attached to a lysine molecule that is cleaved off in the bloodstream, ${ }^{20}$ was first approved in 2007 for Attention-Deficit/Hyperactivity Disorder (ADHD). When the market exclusivity for that indication neared its expiration in February 2012, Shire decreased funding for educational modules on $\mathrm{ADHD}$, while increasing educational grants for binge eating disorder. ${ }^{21}$ In the 18 months before approval of Vyvanse for binge-eating disorder (BED), Shire spent about US \$4 million on increasing physician awareness of BED. ${ }^{21}$

Approval of a previously approved drug for a new indication extends market exclusivity by 3 years. The question of whether BED became a disease to sell a drug led us to investigate whether industry-funded CME on BED was used to promote lisdexamfetamine. We reviewed all Internet CME modules on BED available in 2015 to compare content and key messages between industryfunded and non-industry-funded modules.

\section{Methods}

Online CME modules on BED were identified by searching the terms, "binge eating disorder," combined with "CME" on Google. We limited our results to Web-based CME (in other words, we did not analyze live events). We utilized a qualitative thematic analysis research approach that enables the study of meanings. Using statements of belief, opinion, or putative fact as the units of analysis, thematic analysis is an inductive approach that is used to interpret text data through systematic identification and classification of themes or patterns. Thematic analysis requires identifying, recording, analyzing, and refining key narrative points, called "themes," within a data set of spoken or written material. Our process involved multiple reviewings of modules; extraction and annotation of important teaching points ("themes") that recurred in different modules; repeated refinement of the total list of themes across the data set, analysis of each theme's overt and covert messages, and the use of verbatim quotes to illustrate our analysis.

Both authors viewed several CME activities and established preliminary themes. One author (JJ) reviewed all modules multiple times and took extensive notes, including verbatim quotes, on existing themes and new themes. Both authors added, deleted, and refined themes, based on their recurrence and expression in subsequent modules. Illustrative quotes in our table were selected based on clarity of statement or fluency of expression with respect to particular themes.

Financial conflicts of interest with Shire, the manufacturer of lisdexamfetamine, were identified through the disclosure sections of modules. In addition, all author/presenter names were searched in Dollars for Docs, a database that collates information reported to the Center for Medicare and Medicaid Services' Open Payments, a repository of disclosure information on industry payments to physicians.

\section{Results}

A Google search for Web-based CME on BED generated 13,800 hits. The first 200 results were reviewed, and about 200 additional results on 20 random pages were checked. Twenty-seven ${ }^{22-48}$ different video or text CME modules were identified. No new CME modules appeared after the 37 th result; additional results identified in the 400 results reviewed were duplicates or irrelevant.

All CME modules were accredited by providers certified by the Accreditation Council for Continuing Medical Education. Nine modules 
Figure 1. Number of slides repeated in different online activities on BED. Abbreviation: BED, Binge-Eating Disorder.

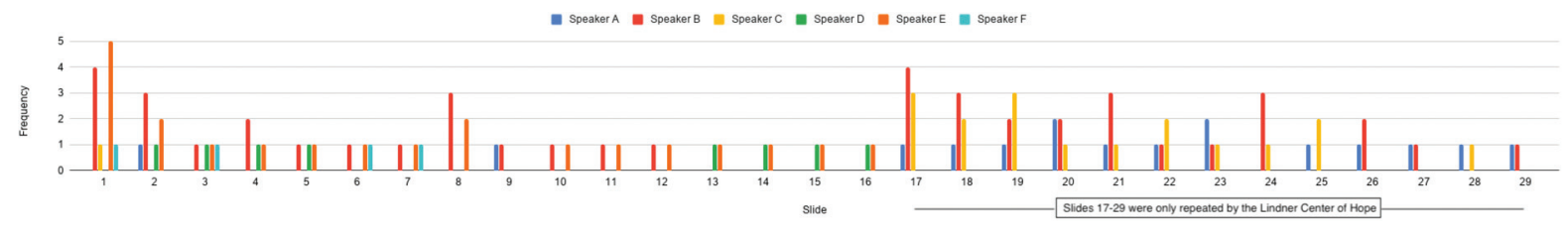

were available on realcme.com, 7 on mycme.com, 4 on provaeducation.com, 3 on medscape.org, and 1 each on primed.com, healio.com, thedoctorschannel.com, and the Peerview Institute for Medical Education, respectively. Three of $9^{22-24} \mathrm{CME}$ modules on BED at realcme.com were listed under weight management; $6^{25-30}$ were listed under psychiatry.

No non-industry-funded CME modules were identified. Shire, the manufacturer of lisdexamfetamine, was the sole listed funder of the programs through educational grants. Fourteen CME modules were published before, and 13 after, the approval of lisdexamfetamine for BED. Sixteen different presenters or authors were identified in 27 CME modules. Eleven speakers presented in 2 or more modules (Speaker E spoke in 7 modules). Five speakers appeared in only 1 module each (Figure 1).

Of the 5 speakers who appeared in only a single module, none reported conflicts of interest with Shire. Seven of 16 presenters reported receiving consulting fees from Shire; these 7 speakers appeared in 2 to 7 modules. Speakers with financial ties appeared more frequently than speakers without ties. Four of 16 presenters (including speakers $\mathrm{A}, \mathrm{B}$, and $\mathrm{C}$ ) were associated with the Lindner Center of HOPE, which received research payments of US $\$ 71,135$ in $2013,{ }^{49}$ US $\$ 164,592$ in $2014^{50}$ and US $\$ 134,137$ in $2015^{51}$ from Shire. -Two of the 4 authors (speakers A and B) associated with the Lindner Center of HOPE also had personal financial conflicts of interest with Shire.

\section{Common Slides}

Twenty-nine slides recurred in at least $2 \mathrm{CME}$ modules. Eight slides were repeated in 5 or more modules (Figure 1). The content of repeated slides included graphics, data tables, and bulleted-list summaries. None of the identical tables or figures used by multiple speakers appeared in sources referenced on the slides. While several graphics used by multiple speakers were found in referenced sources, additional elements were added to the graphics and these alterations were identical in slides used by different speakers.

The most frequently repeated slide, illustrating the prevalence of BED by depicting faces superimposed over a map of the US, appeared in 11 different modules.

Fifteen of 29 slides common to multiple presentations were repeated by 2 different speakers, 11 slides by 3 different speakers, and 3 slides by 4 different speakers. Slide 4 was used by 2 different speakers who presented within the same CME module. ${ }^{31}$ Four modules ${ }^{25-27,32}$ utilized 2 speakers in discussion; in these cases, the slide was attributed only to 1 primary speaker. Three slides that were used by 4 different speakers discussed DSM- 5 criteria for the diagnosis of $\mathrm{BED}$, the prevalence of $\mathrm{BED}$, and characteristics of binge eating. Thirteen of 29 slides were repeated only by staff associated with Lindner Center of HOPE (Figure 1).

\section{Common Themes}

Twelve major themes, present in all modules, were identified:

1. BED is highly prevalent but often a missed diagnosis; clinicians must elicit the diagnosis by questioning patients closely.

2. BED can occur in everyone regardless of age, gender, ethnicity, or weight.

3. BED results in poor quality of life.

4. BED is associated with many other disorders, including obesity, depression, anxiety, substance use disorders, diabetes, dyslipidemia, and metabolic syndrome.

5. Many patients with BED are obese.

6. BED makes losing weight difficult.

7. BED is not a character flaw.

8. BED is related to dopamine dysfunction.

9. BED is a real, treatable disease, and treatment improves lives.

10. Lisdexamfetamine is highly effective for BED. 
11. Topiramate may be effective but its use is limited by substantial adverse effects.

12. Other therapeutic options for BED are inferior to lisdexamfetamine because they do not cause weight loss.

See Table 1 for examples of statements from different modules related to each theme.

\section{Marketing Messages}

Both explicit and implicit marketing messages supporting lisdexamfetamine were identified in these modules. Explicit marketing messages in these modules included the positioning of lisdexamfetamine as a safe, very effective treatment for BED; the positioning of topiramate, serotonin reuptake inhibitors (SSRIs), and other pharmacologic therapies as unsafe, and the framing of psychotherapy as effective but difficult to access. The primary implicit marketing messages in these modules were that BED is associated with obesity, and that lisdexamfetamine was superior to other therapies because only lisdexamfetamine caused weight loss.

None of the 27 modules reviewed mentioned that lisdexamfetamine is an amphetamine with a high potential for abuse. No module mentioned any serious adverse effects of lisdexamfetamine, which include sudden death, stroke, myocardial infarction, hypertension, tachycardia, psychosis, manic symptoms, growth suppression, and peripheral vasculopathy. ${ }^{20}$

In these modules, lisdexamfetamine appeared safe. A typical statement regarding side effects was that the "safety profile was very similar to use of lisdexamfetamine for ADHD, which is usually very well tolerated." ${ }^{11}$ Activities that mentioned any adverse effects at all mentioned only decreased appetite, dry mouth, headache, and insomnia.

Lisdexamfetamine was presented as a highly effective drug for BED, even in modules released before approval for BED. For example, a speaker in 1 module dated before approval of lisdexamfetamine for BED stated: "lisdexamfetamine has more recently been studied with very positive results ... the result has been so positive." ${ }^{33}$ Another stated: "We don't get results like this in many studies. This is incredibly positive data." ${ }^{32}$

Besides lisdexamfetamine, speakers presented all other therapies for treating BED and obesity in a negative light, including topiramate, zonisamide (an anticonvulsant), duloxetine and other SSRIs, antiobesity medications, and psychological therapies.
Topiramate was highlighted for negative attention. For example, 1 speaker stated: "One long-term study of topiramate had a duration of 42 weeks and showed continued improvement in binge eating disorder and weight loss; however, the discontinuation rate for adverse events in this study was high." ${ }^{34}$ Another stated that: "Studies of topiramate have reported superiority over placebo with regard to reductions in binge frequency and weight loss. Topiramate is associated with side effects (eg, paresthesias, dry mouth, headache, dyspepsia, and cognitive impairment) that may have contributed to high dropout rates in some trials. It should be noted that topiramate is considered pregnancy category D and has been associated with increased risk for fetal defects." 22

Cognitive behavioral therapies (CBT) and psychotherapy were presented as effective but difficult to access. For example, 1 presenter stated that psychotherapy is "not commonly available." ${ }^{35}$ The same module stated that: "identify[ing] someone who really knows manual-based approaches such as CBT are hard to find." 35

Every CME module covered obesity and metabolic syndrome. Thirteen modules with 8 different speakers associated binge-eating disorder with weight gain or obesity. ${ }^{22,23,29,31,33-42}$ Messages linking obesity to BED included: "BED is associated with severe obesity." "M1 "Most people presenting for treatment for BED are obese." 36 $\mathrm{BED}$ "is frequently associated with other medical and psychological comorbidities, including obesity." 38

Speakers also stressed the importance of losing weight. A typical statement was, "The weight issue is very important." ${ }^{\prime 35}$ Speakers regularly mentioned that lisdexamfetamine caused weight loss, ${ }^{31,34,35,42}$ and 9 modules noted that pharmacologic and nonpharmacologic therapies for BED other than lisdexamfetamine are ineffective for weight loss. ${ }^{22,28,31-35,37,43}$ Modules stated, for example, that psychotherapy does not "really result in weight loss" 35 or does not lead to "meaningful weight loss". ${ }^{28}$ Other examples include:

"... there is no change in BMI with duloxetine" 32

"So antidepressants have been shown to be somewhat or modestly effective for reducing binge eating over the short term, but they're not usually associated with significant weight loss" 43

"Chronic use of SSRIs is associated with weight gain". 35 
BED is highly prevalent but often a missed diagnosis; clinicians must elicit the diagnosis by questioning patients closely. (8 modules, 7 speakers)

- "These patients often present for weight loss programs or with other comorbidities, such as depression, anxiety, or substance use, but the eating disorder frequently goes undiagnosed" (45).

- "BED hides within previously diagnosed conditions" (46).

- "Pushing a little further can elicit a diagnosis for BED" (32).

- "That it is a hidden disorder that people do not report because they feel such shame, or they do not even know they are doing it" (39).

- "Patients with BED often display discomfort, shame, or guilt regarding their weight and eating behavior. Many patients with BED are secretive about their binge eating and attempt to conceal their behaviors" (22).

- "When you ask these questions to people with binge eating disorder, they often resonate, and people will answer affirmatively when they're specifically probed about their symptoms" (43).

- "Just go one step further and ask that question about uncontrolled eating, and many times you will have a revelation at that patient visit" (40).

- “... probe a little bit... Ask: 'Kendra, tell me a little bit about the eating at night”” (41).

BED can occur in anyone regardless of age, gender, ethnicity, or weight. (12 modules, 5 speakers)

- "BED is a disorder that affects everyone. It causes a lot of suffering across many groups of people" (41).

- "BED can occur. . . in people of any size, weight, and shape" (31).

- "BED occurs across all weight categories" (31).

- "BED is found across all weight, age categories, as well as all ethnic and racial groups in United States" (37).

- "Unlike anorexia nervosa and bulimia, BED appears to affect men and women at similar rates and has been identified across diverse community and clinical samples, ethnicities, and racial backgrounds" (25).

- "... binge eating is more common than previously recognized, occurring in $2.6 \%$ of US adults, in both men and women, and in members of all ethnic/racial groups" (45).

- “... of the eating disorders, BED occurs more in men" (33).

- "BED is the most prevalent eating disorders in males" (47).

- "You may be surprised to hear that almost half are male and actually the gender distribution is far less skewed with BED than it is with anorexia nervosa and bulimia nervosa, where it seems to occur more commonly among females" (29).

- "Like other eating disorders, binge eating disorder affects women more often than men, but binge eating disorder is more common in men than other eating disorders" (43).

- "It is a common problem for both men and women" (48).

BED results in poor quality of life. (7 modules, 5 speakers)

- "The quality of life is impaired" (35).

- "... binge eating disorder is associated with reduced quality of life and impairment in functioning that is actually comparable to that seen in people with bulimia nervosa" (40).

- “... BED is associated with distress, reduced quality of life, and role impairment comparable to that seen with bulimia nervosa” (43).

- "Kids as young as 5 years old are already being stigmatized for overweight. They have poor quality of life. They rated as poor as children with cancer" (34).

- "With all patients with BED, there is an increased risk for them to suffer psychological distress, interpersonal problems, and some role impairments. There have been some reports on elevated suicidality" (27).

- "Many people with BED have additional thoughts about the behavior and these thoughts can be very distracting and it impairs people's ability to function optimally" (44).

- "BED is a disorder that affects everyone, that causes a lot of suffering across many different groups of people" (41).

BED is associated with many other disorders, including obesity, depression, anxiety, substance use disorders, diabetes, dyslipidemia and metabolic syndrome. (7 modules, 7 speakers)

- "Patients with this disorder may have greater impairment and poorer physical health, for example, worsened metabolic profiles, more rapid weight gain, and a worse response to weight loss treatment" (45).

- "Beyond obesity, medical concerns such as metabolic syndrome and diabetes are also frequently associated with BED, leading to a reduced quality of life and impairment in functioning" (25).

- "BED is associated with a huge amount of co-morbidities" (23).

- "As noted, mood and anxiety disorders are commonly comorbid with BED...Presentations that suggest depression or anxiety should increase suspicion for BED" (22).

- "Most clinicians in psychiatric space probably have a number of patients with BED who have presented them with comorbidity, depression, or anxiety - and questions about binge eating have never come up" (29).

- “... BED often co-occurs with other psychiatric and medical disorders, in particular, mood disorders, and that includes both depressive and bipolar disorders, anxiety disorders, substance use disorders, impulse control disorders, including attention deficit hyperactivity disorder, and BED also co-occurs with obesity, and that includes severe obesity, as well as possibly metabolic syndrome"(43).

- "Subjects showed increased frequency of anxiety disorders, substance-related disorders, depressive symptoms, trait anxiety, and higher external and emotional eating scores than subjects without BED” (42).

Many patients with BED are obese. (13 modules, 9 speakers)

- "BED is associated with severe obesity" (31).

- "Most patients with BED are obese" (33).

- "Most people presenting for treatment for BED are obese" (36). 
- "It is associated with increased risk for obesity" (41).

- "It is frequently associated with other medical and psychological co-morbidities, including obesity" (38)

- "... BED does not automatically equate to obesity, but it definitely increases the risk of obesity - by about $75 \%$ in 1 large study" (39).

- "Studies reveal overlaps among appetitive traits that likely increase risk for binge eating symptoms and excess weight gain ... In this sense, the disorder is a biologically based subtype of obesity. The proneness to bingeing behaviors can lead to hyperreactivity to the hedonic properties of food" (45).

- "Overweight or obese BED patients are more likely to gain weight more rapidly" (23).

- "The presence of binge eating episodes can lead to weight gain, even when patients engage in food restriction during the rest of the day" (22).

- "Recent substantial weight gains, history of weight fluctuation or current reports of inability to lose weight those are things that need to be monitored and looked at for possible medical reasons as well as they signal for presence of this behavior in psychiatry problem" (37).

- "I think there are clear red flags that determine when we definitely should ask about binge eating - most importantly, whenever somebody presents with a weight problem" (40).

- "Weight issue is very important" (35).

- "Let me talk about a clinical case study: Sally, a White 40 year old woman, who is not losing weight despite being on repeated diets. That alone should clue us in that we need to ask about eating behaviors" (29).

BED makes losing weight difficult. (2 modules, 2 speakers)

- "These red flags include the presence of excess weight or obesity; a history of weight fluctuations, rapid weight gain, and difficulty losing weight" (45).

- "If you can stop binge eating, you can stabilize weight gain" (36).

BED is not a character flaw. (8 modules, 3 speakers)

- "BED is not a sign of weakness or character flaw. It is a medical condition" (36).

- "...I believe we must convince our patients that this is a disease; this not a character flaw" (39).

- "Educate the patient about BED - that it is a medical condition. It is not something that they are choosing to do or is a character flaw" (32).

- "It is not a character flaw. It is not a personal failure" (40).

- "We need to provide patients with education. Not only with information but with validation. How common it is and that it is not a character flaw" (25).

- "I also tell them that binge eating disorder is a distinct medical condition that's classified in the DSM-5, and this can help patients realize that they have a disorder rather than a personal weakness" (43).

- "BED is a diagnosable psychiatric disorder. This is a serious and formal disorder. It is not about willpower" (23).

- "BED is a diagnosable psychiatric disorder. This is a serious mental and behavioral problem. It is not about weakness or it is not about lack of will power" (31).

BED is related to dopamine dysfunction. (4 modules, 4 speakers)

- "BED is associated with dopamine dysfunction while controlling for obesity" (27).

- "Basic research into the neurobiological mechanisms of BED has implicated different neurotransmitters and systems, with varying degrees of evidence. Potentially involved neurotransmitter systems include, among others: dopamine" (22).

- "Studies in humans and animal models suggest a role for dopamine in the pathophysiology of BED" (30).

- "It has been demonstrated that repeated stimulation of dopamine-containing neurons in the midbrain that project to the striatum is associated with the development and maintenance of binge eating" (24).

BED is a real, treatable disease, and treatment improves lives. ( 7 modules, 4 speakers)

- "BED is treatable" $(27,28)$.

- "Treatments exist and include evidence-based forms of psychotherapy and medications" (44).

- "BED is a diagnosable disorder and a treatable disorder" (37).

- "BED is a diagnosable and treatable disorder" (38).

- "There are also some great self-help books out there so that they gain more of an understanding of the disorder and they get more empowered in the management of their own lives. Many times when they do that, it is not just the binge eating that improves, and it is not even the other chronic medical conditions" (39).

- "When you identify BED and treat this patient effectively, the outcome can be outstanding, and you can really make a strong difference in patients' lives by preventing serious morbidity and just helping them to live better, live happier, and be more in control of their own lives" (40).

Lisdexamfetamine is effective for BED. (7 modules, 5 speakers)

- “. . lisdexamfetamine is the only agent to my knowledge with FDA indication for moderate to severe BED" (31).

- "We don't get results like this in many studies. This is incredibly positive data" (32).

- "Once you move up to $50,70 \mathrm{mg}$ [of lisdexamfetamine], then you see very impressive results" (25).

- "Like lisdexamfetamine has more recently been studied with very positive results. . . the result has been SO positive" (33).

- "Pharmacotherapies are widely available and they are not difficult to use. Because of this, this seems to be a very good model for treating BED... if they are not successful you can think about adding structured psychotherapy at that point" (35).

- "It improved people's ability to inhibit their eating behavior when they wanted to. The drug also reduced hunger. And finally, lisdexamfetamine also reduced the obsessive-compulsive features of binge eating. So people spent less time thinking about eating or having urges to binge eat, and they felt more in control of their eating behavior" (43).

- "Both of these studies showed VERY positive results for lisdexamfetamine for BED" (34). 
Topiramate and other treatments for BED cause adverse effects. (5 modules, 5 speakers)

- "Topiramate has been shown to be effective but complicated by high discontinuation rate and side effects" (31).

- "... [topiramate] has been shown to be effective in both binge eating episodes as well as weight loss. The downside being some high discontinuation rates due to side effects" (33).

- "Similarly, you will see effectiveness that was shown from the sibutramene. However, this medication has been withdrawn from the market" (33).

- "One long-term study of topiramate had a duration of 42 weeks and showed continued improvement in BED and weight loss; however, the discontinuation rate for adverse events in this study was high" (45).

- "Topiramate is associated with side effects (eg, parathesias, dry mouth, headache, dyspepsia, and cognitive impairment) that may have contributed to high drop out rates in some trials. It should be noted that topiramate is considered pregnancy category D and has been associated with increased risk for fetal defects" (22).

- "More limited evidence describes similar outcomes with zonisamide, including greater reductions in binge frequency and body weight compared to placebo, as well as high rates of discontinuation" (22).

- “... topiramate has been shown to be effective for reducing binge eating and also effective for weight loss, but there are high discontinuation rates with the compound because of side effects" (31).

- "The anti-obesity agent, orlistat, has been studied in binge eating disorder as well. . .there's a high discontinuation rate with this drug because of side effects" (43).

- "Lipase inhibitors or orlistat have been looked at. . It just prevents fat absorption. It does produce weight loss but no significantly effect on binge eating. It is typically not tolerated - well tolerated" (31).

Other therapeutic options for BED are inferior options because they do not produce weight loss. (9 modules, 5 speakers)

- "Anti-depressants have been studied. Their overall effect on BED has been modest. They do not significantly produce weight loss and their tolerability varies among different types of anti-depressants" (31).

- "...there is no change in BMI with duloxetine" (32).

- "Specialized psychotherapies are generally not effective for weight and obesity" (32).

- "In anti-depressant realm, there has been some modest effect on binge eating episodes, but no significant effect on weight loss itself" (33).

- "So antidepressants have been shown to be somewhat or modestly effective for reducing binge eating over the short term, but they're not usually associated with significant weight loss" (43).

- "Psychological therapies, however, do not produce weight loss without a complementary weight-reduction or anti-obesity intervention" (45).

- "Overall, studies of SSRIs in BED have reported greater reductions in binge eating compared to placebo, although weight reduction was modest in most trials" (22).

- "Chronic use of SSRIs is associated with weight gain" (35).

- "How well do these [nonpharmacological] things work? Do they work on binge eating? Well yeah, they work very well on binge eating. . . but the problem is that they don't really result in weight loss" (35).

- "Help manuals alone or with guidance from therapies may benefit some people with BED but should be only considered as first step in treatment. But not probably effective for many people. . They don't produce clinically meaningful or sustain weight loss" (28).

- "The anti-obesity agent, orlistat, has been studied in binge eating disorder as well, but the results have been mixed. It's really not clear whether or not this compound reduces binge eating behavior, and in some studies, there was weight loss and other studies there wasn't" (43).

- "The anti-obesity medication, sibutramine, has been studied in several randomized controlled trials in people with binge eating disorder, where it's been shown to be effective for reducing binge eating behavior and also effective for weight loss. But this compound has been withdrawn from the market because of safety concerns" (43).

- "Unfortunately, CBT and some of other psychological treatments that are available in special centers the do not seem to produce weight loss unless, of course, there is complementary weight loss intervention or anti-obesity medication or antiobesity intervention provided in adjunct" (34).

- "Other agents such as SSRIs or anti-depressants appear to have some benefit for reducing BE but unfortunately they do not produce any weight loss" (37).

BED, Binge-Eating Disorder; SSRI, serotonin reuptake inhibitors; FDA, Food and Drug Administration ; DSM5, Diagnostic and Statistical Manual of Mental Disorders.

See Table 1 for more examples of statements related to each theme.

\section{Condition Branding Binge-Eating Disorder}

Industry sponsorship affects prescribing; 1 study $^{52}$ of 160 German physicians found that physicians who avoided sponsored CME prescribed more generic and fewer branded drugs than physicians who participated in sponsored CME.

CME is particularly important for disease awareness, or "condition branding," a marketing technique in which a company creates, adopts, or redefines a condition and then develops perceptions of that condition along with marketing a treatment. ${ }^{53}$ Examples include premenstrual dysphoric 
disorder, depicted as a more serious form of premenstrual syndrome to sell Sarafem (fluoxetine), which was repackaged Prozac (fluoxetine). ${ }^{4}$ Social anxiety disorder was created to create a marketing niche for Paxil (paroxetine), a serotonin reuptake inhibitor that entered a market crowded with related drugs for depression. ${ }^{53-55}$ Hypoactive Sexual Desire Disorder (HSDD) was created to sell a medication that purportedly boosted libido in women. ${ }^{4}$

Psychiatric disorders are particularly fertile ground for condition branding, because no lab or imaging tests can confirm psychiatric diagnoses. According to an industry article, condition branding is characterized by "[E]levating the importance of an existing condition and "developing unmet market need." ${ }^{55}$ Statements supporting the first condition include:

"Many people with binge eating disorder have additional thoughts about the behavior and these thoughts can be very distracting and it impairs people's ability to function optimally"44

"with all patients with binge eating disorder, there is an increased risk for them to suffer psychological distress, interpersonal problems, and some role impairments. There have been some reports on elevated suicidality". ${ }^{27}$

Statements supporting "developing unmet market need" include many claims that BED is highly prevalent, underdiagnosed, and can occur in everyone regardless of age, gender, weight, and ethnicity. For example, 1 speaker stated that BED “... is found in men, it is found in women, it is found across ethnic and racial minority groups" and can occur ". . . in people of any size, weight, and shape." 31

BED was included in appendix B ("Criteria Sets and Axes Provided for Further Study") of the DSM-IV, published in $1994 .{ }^{56}$ However, it was diagnosable only using the catch-all category of "eating disorder not otherwise specified." In DSM-5, the American Psychiatric Association (APA) officially included BED and also loosened the frequency and duration criteria: while DSMIV required symptoms to occur at least 2 days a week for 6 months, DSM-5 required only 1 day a week for 3 months. Current DSM criteria for binge eating disorder are 1) recurrent and persistent episodes of binge eating, 2) binge eating episodes associated with 3 or more of the following: eating much more rapidly than normal; eating until feeling uncomfortably full; eating large amounts of food when not feeling physically hungry; eating alone because of being embarrassed by how much 1 is eating; feeling disgusted with oneself, depressed, or very guilty after overeating, 3) marked distress regarding binge eating, and 4) absence of regular compensatory behaviors such as purging. Severity of the disorder is based on the number of episodes of binge eating. ${ }^{57}$

\section{Discussion}

These modules create a sense of urgency regarding an invented "unmet medical need." Linking BED to other conditions increases the population eligible for treatment. It is implied that treating BED will help other psychologic disorders, although it is far more likely that treating anxiety or depression will help binge-eating symptoms than that treating BED will mitigate psychologic symptoms.

Although we sought to determine whether industry-funded CME was used to promote binge eating disorder, we discovered that CME is being used to position lisdexamfetamine as a diet pill. Although lisdexamfetamine was approved to treat $\mathrm{BED}$, it has never been approved by the Food and Drug Administration (FDA) for weight loss. In fact, use for weight loss is specifically contraindicated on the label. ${ }^{20}$

The indirect promotion of lisdexamfetamine as a diet pill was obvious in the CME modules we analyzed. Although speakers never specifically recommended lisdexamfetamine as a weight loss drug, speakers regularly noted that lisdexamfetamine caused weight loss, while dismissing other options because they do not cause weight loss. Although lisdexamfetamine has never been approved for weight loss, some CME modules on BED were listed under weight management on CME portals.

Besides promoting lisdexamfetamine for weight loss, a contraindicated use, the fact that lisdexamfetamine was presented as an effective treatment for BED before the drug's approval for BED also represents promoting an unapproved use.

The repetition of many novel slides by many different speakers in different "educational" modules suggests the use of a company-created promotional slide deck. Companies often distribute a set of slides to paid speakers to convey specific marketing messages (see supplemental material for repeated slides). Although speakers who work together (presumably the case at Lindner Center of HOPE) may well share slides, it is implausible that speakers at 
different sites independently developed identical slides.

Lisdexamfetamine is presented as a safe therapy in these CME modules. Serious harms associated with lisdexamfetamine were minimized or omitted in every module. The claim that lisdexamfetamine is safer than other drugs used for BED is not backed by evidence.

Widely prescribed between the 1940s and the 1970s as a weight loss drug, amphetamines caused an epidemic of stimulant abuse, and the FDA subsequently prohibited the use of amphetamines for weight loss. ${ }^{58}$ The fen-phen fiasco in the 1990s, during which many people developed irreversible pulmonary hypertension from a combination of phentermine (an amphetamine) and fenfluramine (a serotonergic drug subsequently removed from the market in 1997) created an unfriendly regulatory atmosphere for weight loss drugs, especially those containing amphetamines. The regulatory atmosphere may be changing: in 2012, Qsymia, a combination of phentermine and topiramate (an anticonvulsant), became the first antiobesity drug to be approved in 12 years. ${ }^{59}$

Nonetheless, lisdexamfetamine has never been approved by the FDA as a weight loss drug. The CME modules analyzed seem to be part of an offlabel marketing campaign to market lisdexamfetamine as a diet drug.

\section{Limitations}

Using a thematic analysis approach, we attempted to reverse-engineer a list of marketing messages associated with lisdexamfetamine. However, marketing messages are proprietary information and we cannot confirm the accuracy of specific planned marketing messages without access to Shire's internal communications. We were also unable to assess the success or failure of this promotional campaign on the sales of lisdexamfetamine.

\section{Conclusion}

Our study adds to the growing literature showing that industry-funded CME contains marketing messages that favor sponsors' drugs. Our analysis of CME modules on BED, all which were solely funded by the manufacturers of lisdexamfetamine, reveal many marketing messages that advantage lisdexamfetamine over other treatment options, even psychotherapy, arguably the best and certainly the safest option. The omission of life-threatening adverse effects of lisdexamfetamine from every module may give physicians a distorted sense of the risk-benefit ratio of using lisdexamfetamine. Shire seems to be using CME to promote lisdexamfetamine as a weight-loss agent, an off-label claim.

Many aspects of these modules violate standards against commercial bias in CME. This raises serious questions about the ability of CME accreditors and the Accreditation Council on Continuing Medical Education to detect and neutralize commercial bias in CME. Subtle marketing messages may escape detection by CME accreditors and audiences, and these messages may powerfully influence therapeutic choices. The current accreditation system is inadequate. Physicians and other prescribers should avoid industry-funded continuing education, which is really marketing masquerading as education. Industry-funded CME is a form of promotion and should be regulated as marketing.

The authors wish to thank Alycia Hogenmiller and Sophie Krensky for help with manuscript preparation.

To see this article online, please go to: bttp://jabfm.org/content/ 33/2/240.full.

\section{References}

1. Orlowski JP, Wateska L. The effects of pharmaceutical firm enticements on physician prescribing patterns: there's no such thing as a free lunch. Chest 1992;102:270-3.

2. Bowman MA. The impact of drug company funding on the content of continuing medical education. J Contin Educ Health Prof 1986;6:66-9.

3. Carlat D. Dr. Drug Rep. New York Times Magazine. November 17, 2017. https:/www.nytimes.com/2007/ 11/25/magazine/25memoir-t.html. Accessed July 21, 2016.

4. Meixel A, Yanchar E, Fugh-Berman A. Hypoactive sexual desire disorder: inventing a disease to sell low libido. J Med Ethics 2015;41(10):859-62.

5. Fugh-Berman A, Hogenmiller A. CME stands for commercial medical education: and ACCME still won't address the issue. J Med Ethics 2015.

6. Bowman MA, Pearl DL. Changes in drug prescribing patterns related to commercial company funding of continuing medical education. J Cont Educ Health Prof 1988;8:13-20.

7. Anonymous. Drug company leader says, end industry support of CME. Bioethics Forum. The Hastings Center, Garrison, NY. 2010. https://www. thehastingscenter.org/drug-company-leader-says- 
end-industry-support-of-cme/. Accessed July 21, 2016.

8. Steinman MA, Bero LA, Chren MM, Landefeld CS. Narrative review: the promotion of gabapentin: an analysis of internal industry documents. Ann Intern Med 2006;145:284-93.

9. Fugh-Berman A, Melnick D. Off-Label promotion, on-target sales. PLoS Medicine 2008;5 (10):e210.

10. Thomas K. Shire, maker of binge-eating drug vyvanse, first marketed the disease. The New York Times. February 24, 2015. https://www.nytimes. com/2015/02/25/business/shire-maker-of-bingeeating-drug-vyvanse-first-marketed-the-disease.html.

11. Cosgrove L, Krimsky S, Wheeler E, Katz J, Greenspan S, DiPentima N. Tripartite conflicts of interest and high stakes patent extensions in the DSM-5. Psychother Psychosomatics 2014;106-13.

12. Polivy J, Herman CP. Causes of eating disorders. Annu Rev Psychol 2002;53:187-213.

13. Healy M. Is binge eating a psychiatric disorder? Los Angeles Times. November 23, 2009. https:// www.latimes.com/health/la-he-binge23-2009nov23story.html.

14. Frances A. Saving normal: an insider's revolt against out-of-control diagnosis, DSM-5, big pharma, and the medicalization of ordinary life. New York, NY: HarperCollins; 2013.

15. Frances AJ, Widiger T. Psychiatric diagnosis: lessons from the DSM-IV past and cautions for the DSM-5 future. Annu Rev Clin Psychol 2012;8: 109-30.

16. Thomas JJ, Koh KA, Eddy KT, et al. Do DSM-5 eating disorder criteria overpathologize normative eating patterns among individuals with obesity? J Obes 2014;320803.

17. Hom PH, Giles TR. Performance-based intervention for non-purging bulimia: some implications for the treatment of binge eating and obesity. Psychol Health 1991;5:183-91.

18. Krysanski VL, Ferraro FR. Review of controlled psychotherapy treatment trials for binge eating disorder. Psychol Rep 2008;102:339-68.

19. Shire Annual Report 2014. http://investors.shire. $\mathrm{com} / \sim / \mathrm{media} /$ Files/S/Shire-IR/agm-documents/ agm-2015/shire-annual-report-2014-26-03-15.pdf. Accessed July 21, 2016.

20. Vyvanse Important Safety Information. 2018. https:// www.shireregistration.com/vyvanse_savings_link. Accessed July 21, 2016.

21. Transparency Disclosures. https://www.shire.com/ who-we-are/how-we-operate/transparency-disclosures. Accessed July 20, 2016.

22. Coughlin J, Guarda A, Bello NT. Patient challenge identifying and managing binge eating disorder (Gary). Baltimore, MD: Johns Hopkins University School of Medicine. Released 30 April 2015. Expires 29 April 2017. http://www.realcme.com/ cms/node/598352?utm_source=activitylist\&utm_ medium=web\&utm_content=all\&utm_campaign. Accessed September 23, 2019.

23. Grilo CM. Webinar 1: getting to know, screen, and diagnose for binge eating disorder in practice. Released 17 March 2015. Expires 31 March 2016. Jackson Township, NJ: American Academy of CME, Inc. PeerXChange. http://realcme.com/cms/ node/589633?utm_source=activitylist\&utm_medium= web\&utm_content=all\&utm_campaign. Accessed September 23, 2019.

24. Guarda A, Coughlin J, Bello NT. A diagnostic challenge in binge eating disorder (Beth). Baltimore, MD: Johns Hopkins University School of Medicine. Released 14 July 15. Expires 13 July 2017. http://realcme.com/cms/node/609400?utm source=activitylist\&utm_medium=web\&utm_content= all\&utm_campaign. Accessed September 23, 2019.

25. Berens D, Cummings TS. Binge eating disorder: are we missing it? Rancho Mirage, CA: Annenberg Center for Health Sciences at Eisenhower. Released 02 July 2015. Expires 9 June 2016. http://realcme. com/cms/node/603524. Accessed September 23, 2019.

26. Cummings TS, Berens D. Cases in Binge Eating Disorder. Rancho Mirage, CA: Annenberg Center for Health Sciences at Eisenhower. Released 28 July 2015. Expires 28 July 2016. http://www.realcme. com/cms/node/611407. Accessed September 23, 2019.

27. Grilo CM, Pertschuk M. Webinar 4: examining a multidisciplinary approach to managing binge eating disorder. Jackson Township, NJ: American Academy of CME, Inc. PeerXChange. Released 19 May 2015. Expires 9 June 2016. http://realcme. com/cms/node/600751?utm_source=activitylist\&utm_ medium=web\&utm_content=all\&utm_campaign . Accessed September 23, 2019.

28. Keck PE. Webinar 3: emerging strategies in the management of bed the role for pharmacologic and non-pharmacologic approaches. Jackson Township, NJ: American Academy of CME, Inc. PeerXChange. Released 30 April 2015. Expires 19 May 2016. http://www.realcme.com/cms/node/598332?utm source=activitylist\&utm_medium=web\&utm_content= all\&utm_campaign. Accessed September 23, 2019.

29. Citrome LL. Webinar 2: translating and incorporating the new DSM-5 diagnostic criteria for BED into practice. Jackson Township, NJ: American Academy of CME, Inc. PeerXChange. Released 07 April 2015. Expires 20 April 2016. http://realcme. com/cms/node/593069?utm_source=activitylist \& utm_medium=web\&utm_content=all\&utm_campaign. Accessed September 23, 2019.

30. Guarda A, Coughlin J, Bello NT. Recognizing Eating Disorders in Routine Clinical Care (Jackie). Baltimore, MD: Johns Hopkins University School of Medicine. Released 07 April 2015. Expires 6 April 2017. http://www.realcme.com/cms/node/ 
593045?utm_source=activitylist\&utm_medium=web\& utm_content=curricula\&utm_campaign. Accessed September 23, 2019.

31. Grilo CM, Keck PE, Joy EA. The ABCs of BED: what every practicing family physician should know. Jackson Township, NJ: American Academy of CME, Inc. PeerXChange. Released 20 November 2015. Expires 20 November 2016. http://www. mycme.com/the-abcs-of-bed-what-every-practicingfamily-physician-should-know/activity/3471/. Accessed September 23, 2019.

32. Vega CP, Cummings TS. Binge eating disorder: what, when, why, and how? Boston, MA: Pri-med. Released 12 December 2014. Expires 12 December 2015. http://www.pri-med.com/online-education/ webcast/bingeeatingdisorderwhatwhenwhyandhow. aspx. Accessed 13 July 2016.

33. Cummings TS. Recent changes in the diagnosis and management of binge eating disorder. Mason, $\mathrm{OH}$ : Prova Education, Linder Center of HOPE. Released 27 November 2014. Expires 3 March 2015. http://www.provaeducation.com/CME_ EventGroup.asp? eventGroupCode=810N20D4O5. Accessed July 21, 2016.

34. Citrome LL, Grilo CM, Kornstein SG, Wilfley DE. Binge eating disorder: out of the closet and into a new era of diagnosis and treatment. Thorofare, NJ: Vindico Medical Education. Released 30 September 2014. Expires 29 September 2015. https://www. thedoctorschannel.com/view/binge-eating-disordercme-part-1/. Accessed September 23, 2019.

35. Mitchell JE. Update in the management of binge eating disorder: appropriate diagnosis and treatment. Tinton Falls, NJ: Integrity Continuing Education, Inc. Released 05 May 2015. Expires 5 May 2016. http://www.mycme.com/update-in-themanagement-of-binge-eating-disorder-appropriatediagnosis-and-treatment/activity/2419/. Accessed September 23, 2019.

36. McElroy SL. Management of binge eating disorder. Mason, OH: Prova Education, Lindner Center of HOPE. Released 31 December 2014. Expires 31 December 2015 http://www.provaeducation. com/CME_Event.asp? eventCode=290M21M30O36. Accessed July 13, 2016.

37. Grilo CM. The ABC's of binge eating disorder: a primer for primary care practice-Module 5: examining BED management in primary care practice. Jackson Township, NJ: American Academy of CME, Inc. PeerXChange. Released 30 April 2014. Expires 1 May 2015. http://www.mycme.com/theabcs-of-binge-eating-disorder-a-primer-for-primarycare-practice-module-5-examining-bed-managementin-primary-care-practice/activity/1780/. Accessed September 23, 2019.

38. Keck PE. The ABC's of binge eating disorder: a primer for primary care practice-Module 2: identifying and assessing clinical red flags in practice. Jackson Township, NJ: American Academy of CME, Inc. PeerXChange. Released 30 April 2014.
Expires 1 May 2015. http://www.mycme.com/theabcs-of-binge-eating-disorder-a-primer-for-primarycare-practice-module-2-identifying-and-assessingclinical-red-flags-in-practice/activity/1777/. Accessed September 23, 2019.

39. McElroy SL, Vega CP. Improving the management of binge eating disorder: a collaborative treatment approach. New York, NY: Medscape, LLC. Released 02 September 2014. Expires 2 September 2015. http://www.medscape.org/viewarticle/830450_ transcript. Accessed September 23, 2019.

40. Bulik CM, Keck PE, McElroy SL, et al. Recent advances in the management of binge eating disorder. New York, NY: Medscape, LLC. Released 26 March 2014. Expires 26 March 2015. http://www. medscape.org/viewarticle/821956. Accessed September 23, 2019.

41. Grilo CM. The ABC's of binge eating disorder: a primer for primary care practice-Module 1: dispelling misconceptions about BED. Jackson Township, NJ: American Academy of CME. PeerXChange. Released 30 April 2014. Expires 1 May 2015. http://www.mycme.com/the-abcs-ofbinge-eating-disorder-a-primer-for-primary-carepractice-module-1-dispelling-misconceptionsabout-bed/activity/1771/. Accessed September 23, 2019.

42. McElroy SL. Assessing the impact of binge eating disorder: focus on your practice. New York, NY: Medscape, LLC. Released 26 December 2013. Expires 26 December 2014. http://www.medscape. org/viewarticle/818103. Accessed September 23, 2019.

43. McElroy SL. An update on the diagnosis and treatment of binge eating disorder: implications for clinical practice. New York, NY: Medical Learning Institute, Inc, PVI, PeerView Institute for Medical Education. Released 22 September 2015. Expires 21 September 2016. http://www.peerviewpress. com/update-diagnosis-and-treatment-binge-eatingdisorder-implications-clinical-practice?MemberID=\& SpecialtyID=45\&source=OCME $\&$ ProfessionID $=\&$ CountryID $=\&$ Promocode $=700 \&$ specialty $=180 \&$ ProfID=12\#featured-presentations. Accessed July 21, 2016.

44. Keck PE. The ABC's of binge eating disorder: a primer for primary care practice-Module 3: applying patient communication strategies into practice to improve the management of BED. Jackson Township, NJ: American Academy of CME, Inc. PeerXChange. Released 30 April 2014. Expires 1 May 2015. http://www.mycme.com/the-abcs-ofbinge-eating-disorder-a-primer-for-primary-carepractice-module-3-applying-patient-communicationstrategies-into-practice-to-improve-the-managementof-bed/activity/1778/. Accessed September 23, 2019.

45. Citrome LL, Grilo CM, Kornstein SG, Wilfley DE. Binge Eating Disorder: Out of the Closet and Into a New Era of Diagnosis and Treatment. West 
Deptford, NJ: Healio. Released 01 December 2014. Expires 1 December 2015. No longer available online.

46. Wassenaar E. Binge eating disorder: screening and diagnosis. Mason, OH: Prova Education, Lindner Center of HOPE. Released 5 December 2014. Expires 5 December 2015. http://www. provaeducation.com/CME_Event.asp? eventCode= 977L43K25O43. Accessed July 21, 2016.

47. Cummings TS. Binge eating disorder meets DSM5. Mason, OH: Prova Education, Lindner Center of HOPE. Released 31 December 2014. Expires 31 December 2015. http://www.provaeducation.com/ CME_Event.asp? eventCode=927N55M30O35. Accessed July 21, 2016.

48. Keck PE. The ABC's of Binge Eating Disorder: A Primer for Primary Care Practice-Module 4: Evolving Approaches in the Diagnosis and Treatment of BED. Jackson Township, NJ: American Academy of CME, Inc. PeerXChange. Released 30 April 2014. Expires 1 May 2015. http://www.mycme.com/the-abcs-of-binge-eatingdisorder-a-primer-for-primary-care-practicemodule-4-evolving-approaches-in-the-diagnosis-andtreatment-of-bed/activity/1779/. Accessed September 23, 2019.

49. Shire. Shire US research payments. https://www. shire.com/-/media/shire/shireglobal/shirecom/pdffiles/ transparency/2013-shire-us-research-payments.pdf?la= en. Published 2013. Accessed July 20, 2016.

50. Shire. Shire US research payments. https://www. shire.com/-/media/shire/shireglobal/shirecom/pdffiles/ transparency/2014-shire-us-research-payments.pdf?la= en. Published 2014. Accessed July 20, 2016.

51. Shire. Shire US research payments. https://www. shire.com/-/media/shire/shireglobal/shirecom/pdffiles/ transparency/2015-shire-us-research-payments.pdf?la= en. Published 2015. Accessed July 20, 2016

52. Lieb K, Scheurich A. Contact between doctors and the pharmaceutical industry, their perceptions, and the effects on prescribing habits. PLoS ONE 2014;9:e110130.

53. Angelmar R, Angelmar S, Kane L. Building strong condition brands. J Med Market 2007;7:341-51.

54. Lane C. How shyness became an illness: a brief history of social phobia. Common Knowledge 2006;12: 388-409.

55. Parry V. The art of branding a condition. Med Mark Media 2003;38:43-6.

56. American Psychological Association. Diagnostic and statistical manual of mental disorders. 4th ed. Washington, DC: American Psychological Association, 1994.

57. American Psychological Association. Diagnostic and Statistical Manual of Mental Disorders. 5th ed. Arlington, VA: American Psychiatric Publishing; 2013.

58. Rasmussen N. America's first amphetamine epidemic 1929-1971: a quantitative and qualitative retrospective with implications for the present. Am J Public Health 2008;98:974-85.

59. Azebu LM. The FDA's risk/benefit calculus in the approvals of Qsymia and Belviq: treating an obesity epidemic while avoiding another fen-phen. Food Drug Law J 2014;69:87-111, ii-iii. 